\title{
EL SECTOR FINANCIERO EN COLOMBIA TRAS LA IMPLEMENTACIÓN DEL XBRL COMO HERRAMIENTA DE CONTROL
}

Julián Esteban Zamarra Londoño Universidad de Antioquia Colombia

Tania Atehortúa Castrillón Universidad de Antioquia Colombia

Carlos Eduardo Castaño Rios Universidad de Antioquia

Colombia 
Panorama Económico, Vol. 27 - No. 2 (Abril - Junio de 2019), pp. 527-543

Julián Esteban Zamarra Londoño

Tania Atehortúa Castrillón

Carlos Eduardo Castaño Rios

JEL: G20, G28, G29

\title{
El sector financiero en Colombia tras la implementación del XBRL como herramienta de control
}

\section{Resumen}

El Extensible Business Reporting Language - XBRL llegó al sector financiero colombiano a partir de las obligaciones generadas por la Superintendencia Financiera durante los años 2015 y 2016, situaciones que llevaron a que este grupo de entidades fueran las primeras en acercarse de forma amplia a este lenguaje en el país. Este trabajo parte del objetivo de analizar el proceso de implementación de XBRL en el sector financiero colombiano, y para ello, se tomó la experiencia de una entidad representativa en dicho sector a partir de la documentación jurídica, del proceso de convergencia y recolección de información vía entrevista. Se evidencia que a pesar de que ya se implementó este proceso en las entidades financieras y que se esperan ventajas en el mediano plazo, aún no se tiene la suficiente claridad sobre cómo se facilitará el acceso a la información y los reales beneficios tanto para la entidad reportante como para los interesados en la información.

Palabras clave: XBRL, Sector Financiero, Reportes Contables, Calidad de la información contable.

\section{Le secteur financier en Colombie après la mise en œuvre du XBRL comme outil de contrôle}

\section{Résumé}

Le langage XBRL (Extensible Business Reporting Language) a atteint le secteur financier colombien à partir des obligations générées par la surintendance financière au cours des années 2015 et 2016, ce groupe d'entités étant le premier à aborder largement ce langage dans le pays. Ce travail part de l'objectif d'analyser le processus de mise en œuvre de XBRL dans le secteur financier colombien, et pour cela, l'expérience d'une entité représentative dans ce secteur a été tirée de la documentation juridique, du processus de convergence et de la collecte d'informations via un entretien. Il est évident qu'en dépit du fait que ce processus a déjà été mis en œuvre dans les institutions financières et que des avantages sont attendus à moyen terme, il n'y a toujours pas suffisamment de clarté sur la manière dont l'accès à l'information sera facilité et les avantages réels pour les deux entités. journaliste comme pour ceux qui s'intéressent à l'information.

Mots clés: XBRL, Secteur financier, Rapports comptables, Qualité des informations comptables.

\section{The financial sector in Colombia after the implementation of the XBRL as a control tool}

\begin{abstract}
The Extensible Business Reporting Language (XBRL) reached the Colombian financial sector from the obligations generated by the Financial Superintendence during the years 2015 and 2016, this group of entities being the first to approach this language widely in the country. This work starts from the objective of analyzing the implementation process of XBRL in the Colombian financial sector, and for this, the experience of a representative entity in said sector was taken from the legal documentation, the convergence process and information collection via interview. It is evident that despite the fact that this process has already been implemented in financial institutions and that benefits are expected in the medium term, there is still not enough clarity on how access to information will be facilitated and the real benefits for both the entities. reporter as for those interested in the information.
\end{abstract}

Keywords: XBRL, Financial Sector, Accounting Reports, Quality of accounting information. 


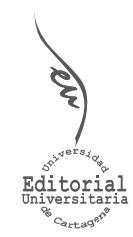

Artículo de Investigación

\section{El sector financiero en Colombia tras la implementación del XBRL como herramienta de control}

INFORMACIÓN DEL ARTíCULO

Recepción del artículo: 07/08/2018

Concepto de evaluación: 23/09/2018

Aceptación del artículo: 03/11/2018
Julián Esteban Zamarra Londoño Universidad de Antioquia, Colombia

Tania Atehortúa Castrillón Universidad de Antioquia, Colombia

Carlos Eduardo Castaño Rios ${ }^{1}$ Universidad de Antioquia, Colombia

\section{INTRODUCCIÓN}

La historia del sistema financiero de cualquier país tiene estrecha relación con las características sociales y económicas del mismo. En Colombia, el primer indicio concreto que se tiene sobre el establecimiento de espacios oficiales como un banco estatal se dio en el Congreso Constituyente de Cúcuta de 1821, pero la idea no prosperó para aquella época (Caballero, 2010). Así mismo, la primera banca comercial surgió a mediados del siglo XIX cuando el país empezó a interactuar fuertemente con el mundo a raíz de las exportaciones agrícolas. Así, poco a poco se fueron estructurando algunas entidades que no prosperaron y que se fueron cerrando casi con la misma rapidez con que se creaban.

No fue sino hasta 1923 cuando la "misión Kemmerer", denominado así por su principal líder el profesor Erick Kemmerer, que el sistema financiero colombiano empezó a estructurarse tal y como se conoce hoy en día. A raíz de la crisis que atravesó el país en 1921, se creó en 1922 por parte del Congreso el Banco de la República y se facultó al gobierno para traer una misión de estudio extranjera para ayudar a reestructurar la economía, la cual fue necesaria para eliminar la carencia de un sistema administrativo y financiero en el país. Como resultado surgió la Superintendencia Bancaria, centro normativo y de vigilancia para todas las entidades bancarias y de crédito (Santos, 2005). Para ese entonces ya existían en Colombia sucursales de algunos bancos extranjeros como el First National Citibank o el Banco Francés. 
Para 1928 se creó la Bolsa de Bogotá para la capitalización de los mismos bancos y de las empresas principalmente mineras. En la primera mitad de los años cincuenta se instituyeron los bancos estatales bajo el objetivo de otorgar préstamos para sectores específicos, siendo estos, por ejemplo, el Banco Popular, Banco Ganadero y Banco Cafetero. Poco después surgieron las corporaciones financieras, fundadas por empresarios que querían invertir en sectores productivos. Para finales de los años setenta surgieron entidades que fueron adquiriendo popularidad como Davivienda, Granahorrar, Conavi, Colpatria, Colmena o Ahorramás. Finalmente, Ocampo (2015) asegura que con la Constitución Política de 1991 el país pudo consolidar una política macroeconómica que favoreció la integración y consolidación del sector financiero.

Desde esta consolidación y hasta ahora, el sistema financiero colombiano ha exhibido una fuerte expansión que se sustenta en el crecimiento de la oferta de servicios y la creciente intermediación, de manera tal que se ha convertido incluso en referente para otros países de la región. Esto se debe, en buena parte a que el sector tiene bases normativas fuertes, así como aseguramiento de sostenibilidad y confiabilidad en entidades como el Banco de la República, la Superintendencia Financiera y Fogafín.

De esta manera, el sector ha adquirido un enorme potencial y relevancia dentro de la economía local, convirtiéndose en un motor para el crecimiento que llega a representar dentro del PIB promedios del 20\% del valor agregado total y un crecimiento medio anual de 5\% en los últimos 10 años (DANE, 2017). Esa situación indica que el sector se expande mucho más rápido de lo que se cree, debido a un fuerte proceso de bancarización e incremento en la intervención financiera y en actividades inmobiliarias y de aseguradoras. Se espera que el sector siga siendo clave en la economía en el sentido de que cada vez está más dinamizado, se amplía la oferta y aumenta la participación en intermediaciones para el desarrollo de negocios locales e internacionales.

No obstante, Hernández (2017) afirma que si bien es cierto que el sistema financiero colombiano es fuerte y podría ser referente, junto con Brasil y Chile, para otros países de Latinoamérica, dadas las condiciones sociales, políticas y económicas del entorno local y, sobretodo, del entorno internacional que atraviesa momentos coyunturales, no ha podido convertirse en un referente global. La principal causa ha sido la inestabilidad institucional que afecta directa o indirectamente el crecimiento del sector a un ritmo aún más acelerado para estar a la altura de industrias como las de Hong Kong, Australia, Estados Unidos y Canadá, aunque está claro que no se han escatimado esfuerzos entre el regulador, supervisor y las entidades financieras que hoy en día se han articulado para mejorar el entorno del sector.

La vía más concreta en la que se pueden evidenciar dichos esfuerzos es la de la del campo regulatorio, puesto que se ha entendido que es necesario controlar las operaciones del sistema financiero debido a la asimetría de la información entre oferentes y demandantes y a las fallas de mercado que existen per se. Mishkin (1996) resalta el inconveniente de las asimetrías de información que afectan el entorno económico y por ende, la estabilidad del sistema financiero.

De esta manera, por el solo hecho de jugar un papel tan importante dentro del desarrollo de cualquier país, es estrictamente necesario que se vigile y controle a dicho sector, de manera que se estructuren guías y normatividades para el desarrollo de negocios y para la revelación de información que sea pertinente para mantener 
un control adecuado. En atención a ello, en la actualidad ya se han desarrollado mecanismos que tratan de eliminar en gran medida dichas asimetrías y se han implementado rápidamente en el sector, debido a su potencial de aplicabilidad y a la importancia del mismo.

Ejemplos de estos mecanismos de mejora son el proceso de estandarización contable en lo que concierne a la regulación de estándares internacionales de información contable y financiera y el uso del lenguaje estándar XBRL para la comunicación de reportes financieros, sin tener en cuenta los requisitos que ya tenían aquellas entidades que guardan relación, por ejemplo, con los mercados norteamericanos en los que las normas están mucho más arraigadas y establecidas.

Actualmente los niveles de solvencia y solidez del sistema financiero que son fruto de su buen desempeño durante los últimos años, dan un voto de confiabilidad sobre la estructura robusta del mismo y su potencial de crecimiento acelerado de manera tal que puede seguir siendo un sector clave para Colombia (Publicaciones Semana, 2015). No obstante, las condiciones económicas en las que se encuentra el mundo hoy en día podrían tener implicaciones sobre la consecución de objetivos ya establecidos con respecto al aumento de participación en el mercado o al mejoramiento de los resultados de la industria. Aspectos como el precio del petróleo y su consecuencia sobre la tasa de cambio tienen enorme incidencia en el desempeño de la industria financiera. Además, los índices de inflación y el leve estancamiento de la economía también pueden tomar relevancia en este sentido, además de las implicaciones que estas situaciones tienen sobre el riesgo de crédito. Bajo un escenario macroeconómico favorable, no hay duda de que el sistema financiero colombiano será más próspero que nunca considerando su excelente desempeño, potencial y capacidad para atender una mayor parte del mercado.

En coherencia con lo anterior, en este artículo se realiza un estudio de caso del proceso de implementación de XBRL en el sector financiero colombiano, planteando reflexiones acerca de la calidad con la cual la información ha sido revelada, el interés y la posibilidad de diferentes usuarios para acceder a ella y las bondades de comparación que puedan haberse extraviado debido a la multiplicidad de taxonomías empleadas para reportar información financiera en el país y globalmente. Las conclusiones giran en torno a la efectividad o no efectividad del objeto del XBRL, la cual está en automatizar, homogenizar y volver comparable el reporte de la información financiera, lo cual se espera se constituya en un aporte de base para abordar tanto el sector financiero como este lenguaje contable para Colombia en línea con las necesidades de sembrar más investigación que recoja las experiencias empíricas para contrastar con la teoría (Castaño, 2016).

\section{IMPLEMENTACIÓN DEL NUEVO MARCO TÉCNICO NORMATIVO CONTABLE Y EL XBRL EN COLOMBIA}

Las Normas Internacionales de Información Financiera NIIF (IFRS por sus siglas en inglés) se han convertido en un hito en la historia contable y financiera que cada vez adquiere mayor importancia en el mundo, fruto del cubrimiento de necesidades básicas de los mercados y, especialmente, de inversionistas interesados en estudiar diferentes opciones para colocar su capital, de modo que exigen por parte de sus potenciales receptores información clara, confiable y, sobretodo, comparable, que les permita analizar y seleccionar las mejores alternativas. 
La situación anterior, unida a un conjunto de necesidades por uniformar los estándares que guían la información de mercados, indujeron a que, inicialmente las grandes entidades financieras del mundo firmaran un acuerdo de normalización con el Comité Internacional de Normas de Contabilidad (IASB por sus siglas en inglés International Accounting Standards Boards) que condujo a que el 17 de mayo del 2000 se produjese la aceptación de las Normas Internacionales de Contabilidad dentro de dicha comunidad. Desde aquel momento, todo el mundo financiero enfocó su atención hacia las normas emitidas para ese entonces por el International Accounting Standards Committee (IASC por sus siglas en inglés) (López \& Zea, 2011).

Concretamente Colombia inició el proceso de normalización hacia los estándares emitidos por el IASB después de la entrada en vigencia de la Ley 1314 de 2009 y las orientaciones que el nuevo Consejo Técnico de la Contaduría Pública emitió para regular la contabilidad en el país. Esta decisión de empezar a planear la implementación de la nueva reglamentación es, sin duda alguna, uno de los más grandes avances en el campo contable nacional, pues existían (y aún quedan algunos rezagos) guías de varias autoridades gubernamentales, tales como los organismos de control -sobre todo las Superintendencias- el Consejo Técnico de la Contaduría Pública (CTCP), Código de Comercio, enlaces tributarios, etc. De modo, que las entidades tenían muchas referencias normativas, siendo el principal y generalmente aceptado en Colombia, el Decreto 2649 de 1993. Sin embargo, en la práctica se había originado una desigualdad en la información contable tan amplia, que generó un escenario en el cual todas estas situaciones jamás hubieran permitido que en Colombia se diera la existencia de un bloque compacto de criterio para reconocer las transacciones (Cano, 2009).

Las normas asociadas al proceso de convergencia crearon tres grupos de entidades que aplicarían en diferentes niveles de rigurosidad los nuevos marcos técnicos normativos contables. El grupo 1, reglamentado inicialmente por el Decreto 2784 de $2012^{2}$, está integrado por las entidades de interés público y aquellas que cotizan en el mercado de valores, dentro de las cuales encajan la mayoría de las organizaciones más relevantes que pertenecen al sector financiero colombiano, y se les exigió aplicar las NIIF Plenas (IFRS Full). La normatividad aplicable para este grupo de empresas está en el Decreto 2483 emitido por el Ministerio de Comercio, Industria y Turismo el 28 de diciembre de 2018, por medio del cual se compilan y actualizan los marcos técnicos de las Normas de Información Financiera NIIF para el Grupo 1 y de las Normas de Información Financiera, NIIF para las Pymes, Grupo 2, anexos al Decreto 2420 de 2015, modificado por los Decretos 2496 de 2015, 2131 de 2016 y 2170 de 2017 , respectivamente, y se dictan otras disposiciones.

Por su parte el grupo 2, reglamentado a través del Decreto 3022 de 2013, fue compuesto por las pequeñas y medianas empresas PYMES y aplican el marco normativo especialmente diseñado para ellas - NIIF para PYMES (IFRS for SME's). Finalmente, el grupo 3 reglamentado por el Decreto 2706 de 2012, está conformado por aquellas empresas de menor tamaño, denominadas microempresas, que aplicarán contabilidad simplificada, siempre que sus ingresos no superen en un año los 6.000 salarios mínimos legales mensuales vigentes en Colombia. 
En lo que concierne al sector financiero y el proceso de convergencia es innegable que su capacidad y la importancia que tienen dentro de cualquier mercado los incita a ser pioneros en un proceso de dichas magnitudes. De hecho, el sector bancario y financiero fue uno de los que mayor avance presentó en la convergencia total a normas internacionales, entre otras cosas porque muchas de estas entidades ya aplicaban alguna normativa fruto de su interacción con mercados extranjeros y principalmente interacciones en el mercado estadounidense que las obligaba, en muchos casos, a presentar informes a la Comisión de Bolsa y Valores de Estados Unidos (SEC por sus siglas en inglés, Securities and Exchange Commission) que para el 2015 permitía una presentación acorde a dichos estándares internacionales.

No obstante los avances significativos, Asobancaria (2012) expresó que el sector financiero todavía afrontaba retos enormes y ajustes necesarios para que el proceso de convergencia fuera exitoso. Por ejemplo, se mencionó la creación o adecuación de plataformas tecnológicas para el tratamiento y reporte de la información, la actualización de guías y manuales de procedimientos que implicaban el uso de mayores recursos tanto en tiempo como en dinero y que exigían un escenario normativo mucho más estable del que se tenía para entonces y cuyo desarrollo era responsabilidad de los organismos reguladores involucrados en la Ley 1314 de 2009.

Precisamente, la Superintendencia Financiera ha adelantado en el país el proceso de acompañamiento e implementación del lenguaje XBRL como mecanismo que facilita la comunicación y comparabilidad de los datos transaccionales reconocidos, puesto que se ha identificado su pertinencia y ajuste con los objetivos de las NIIF, tal y como lo especifica el organismo regulador. Para alcanzar el objetivo, la Superintendencia debió ajustarse a los nuevos estándares de manera que fuesen viables las modificaciones necesarias en pro del éxito de la herramienta comunicativa. La Superintendencia Financiera (2015) reconoce que:

En promedio, las entidades asociadas consideran que requerirán de un período comprendido entre 13 y 24 meses, tanto para aplicar NIIF como para empezar a manejar la taxonomía XBRL. Este lenguaje ha sido utilizado para el desarrollo de una taxonomía de propósito general por parte del IASB, el mismo organismo que desarrolló las NIIF, por lo que se considera adecuada su utilización en Colombia, con un mínimo de ajustes (pág. 12).

La implementación del lenguaje implicó, desde el punto de vista operativo, ciertas inversiones de magnitud considerable que facilitaran la comprensión y utilización de las taxonomías que componen el XBRL para la presentación uniforme de los reportes financieros. Pese a los esfuerzos que un proceso como este acarrea, la Superintendencia Financiera (2015) planteó que los beneficios que se obtienen del mismo son altamente confortables en el sentido de que le permiten al sector tener mayor representatividad en todos los espacios, de forma tal que "permita la utilización de dicha información financiera de manera comprensible, transparente y comparable para cualquier inversionista o usuario de dicha información a nivel nacional e internacional" (pág. 2).

Si bien es cierto que el mercado norteamericano no ha decidido aún implementar las NIIF, la aplicación de este marco normativo y de su representación por medio del XBRL le garantizan a las entidades del país el cumplimento de los mandatos que exige la SEC para presentar informes en el contexto estadounidense, en el que de 
hecho transan las compañías más importantes del mundo y donde se afianzan para la creación de mejores relaciones con todos los grupos de interés y, especialmente, con reguladores e inversionistas.

\section{CONCEPTUALIZACIÓN DEL XBRL}

El eXtensible Business Reporting Language (XBRL por sus siglas en inglés) es un mecanismo o lenguaje de comunicación electrónica estandarizado para la información financiera que surgió en 1998 con el profesional de la contaduría Charles Hoffman como una extensión del XML y que ha sido desarrollado principalmente por el consorcio XBRL International (LEGIS, 2014).

Este sistema suple la necesidad de hacer de los reportes financieros un conjunto de datos mucho más comprensibles y comparables, en la medida que permiten reportar toda la información bajo las mismas etiquetas, procesándose y almacenándose en depósitos electrónicos confiables que permiten, entre otras, reducir la manipulación de los reportes y aumentar la eficiencia en el tratamiento de datos y la eficacia en la comunicación con los agentes de interés; considerando no sólo los reportes externos, sino también reportes internos en las organizaciones que pueden ser utilizados en cualquier parte de la fase de suministro de información financiera.

Las formas tradicionales de intercambio de información en formato HTML como Adobe acrobat, Microsoft Excel, Word o Power Point, no permiten que los datos de los informes, como los estados financieros, puedan ser llevados por un usuario de la información a un software analítico con un nivel razonable de fiabilidad y calidad, aun cuando se tengan, por ejemplo, estructuras definidas de reporte entre la entidad emisora y un órgano regulador. Este problema se presenta porque estos documentos electrónicos no permiten el reconocimiento de características atribuibles a cada dato, como lo son: el tiempo en el cual se presenta una cifra, la moneda y su aproximación o redondeo, la compañía que lo presenta, etc., ya que, si bien los informes financieros pueden presentar encabezados o notas aclaratorias para dar contexto a los datos presentados, en sí mismos carecen de tal contexto.

El XBRL resuelve este problema y proporciona metadatos de alta calidad sobre los hechos y transacciones económicas en los informes financieros en Internet o en intranets. Los datos presentados no tienen sentido a menos que se vean en su contexto y los metadatos proporcionan ese contexto (Debreceny, Felden, Ochocki \& Piechocki, 2009). Estos metadatos son asignados a los datos a través de etiquetas que relacionan atributos como periodo de tiempo, jerarquía de los datos, descripción del concepto, autoridad y marco de regulación contable y financiera, moneda de presentación y otras formas de medida, relaciones de cálculos matemáticos, presentación de los datos en el informe, entre otros.

Por las bondades que el XBRL reporta, en el contexto colombiano la herramienta de comunicación está siendo implementada y aplicada a la par que se empiezan a emitir estados financieros bajo el enfoque de la regulación contable internacional, con el soporte y requerimiento de entidades como el Ministerio de Hacienda y Crédito Público y la Superintendencia Financiera. Para el CTCP (2016), el lenguaje tiene la capacidad de reemplazar los tradicionales sistemas de cuentas que contribuyen en cierta medida a la tergiversación de la información publicada puesto que muchos de ellos son de uso específico para los diferentes sectores de la economía. 
En la actualidad es innegable que la globalización de los mercados y el derrumbe de barreras han incursionado a las organizaciones en un contexto en el que la información que reporte debe permitirle sobresalir en el sentido de que sea mucho más legible, entendible y comparable contra las propuestas o resultados de competidores directos.

La consolidación de los estándares internacionales de información financiera y los esfuerzos de reguladores como la Superintendencia Financiera han permitido dentro del contexto colombiano alcanzar un nivel superior de revelación bajo la metodología de comunicación de los reportes con el sistema XBRL. Este proceso ha sido posible gracias a las experiencias que ya han sido reportadas en otros lugares como Canadá, gran parte de la Unión Europea y los Estados Unidos. Sin embargo, es importante reconocer que hay algunos factores que no facilitan la adopción total del lenguaje puesto que se requieren algunas adecuaciones específicas para el contexto local, tarea en la que ha venido trabajando el regulador para la creación y apropiación de una taxonomía única en todas las organizaciones que estén implementando el nuevo marco técnico normativo contable.

En esa línea, un avance significativo para el adecuado manejo del XBRL en el contexto colombiano ha sido la adopción del marco normativo internacional, pues, aunque el sistema funciona bajo cualquier tipo de estándares es indiscutible que una mayor armonía con la información que circula en la gran parte del mundo también favorece la comparabilidad y homogeneidad de la misma (Superintendencia de Sociedades, 2016). Esta metodología de implementación ha permitido que la comunicación sea mucho más entendible dado que los usuarios de la información analizan más fácilmente los reportes desde el punto de vista que pueden ir haciendo comparaciones organizacionales a medida que realizan dicho análisis y tener una visión mucho más global para realizar, por ejemplo, estudios de mercado y elecciones de inversión. Sin embargo, se puede anotar que en la práctica aún se encuentran diferencias de interpretación y aplicación estándares contables en los diferentes países, sumado a que se hacen adaptaciones a las taxonomías, lo que en definitiva afecta la comparabilidad de la información (Zamara, Atehortúa, \& Sierra, 2017).

No obstante, no queda duda de que el lenguaje facilita la materialización de mayor eficiencia en el uso de recursos y en satisfacción por parte de los agentes de interés que hacen uso de la información y que se caracteriza por un grado elevado de comparabilidad que, de acuerdo con Jensen y Xiao (2001), facilita además la intercambiabilidad a través de plataformas tecnológicas que reducen el tiempo de consulta y análisis y, permiten la toma de decisiones mucho más acertadas y confiables dentro de un proceso que va beneficiando en línea corrida a todas las partes intervinientes.

En este sentido, el hecho de que algunas compañías colombianas emitan informes para su revisión por parte de la SEC es una evidencia del punto de comparabilidad que la información contiene en referencia a otras organizaciones de todo el mundo, dado a la representatividad de la SEC como agente regulador del mercado de valores norteamericano en el que cotizan diversos tipos de compañías. Desde este punto de vista, la información no debería ser incomparable más allá de las extensiones que el lenguaje permite crear dependiendo de las características del sector productivo en el que está inmersa la compañía reportante, puesto que en el momento de creación del mecanismo se entendió la necesidad que surge en algunos sectores sobre hacer referencia a etiquetas exclusivas que no necesariamente son extensibles a otros 
segmentos del mercado pero que seguramente sí son relevantes en un contexto de análisis con una empresa comparable directamente.

Lo anterior es ampliamente extensible al sector financiero que, como ya se ha hecho referencia en los apartados anteriores, se ha visto abocado a implantar en el proceso de convergencia, el conjunto de principios contables y financieros basados en buenas prácticas internacionales, así como el mecanismo de comunicación de la información resultante de la aplicación de dichos principios, siendo estos elementos relevantes dentro de cualquier economía por la constante búsqueda de transparencia y comparabilidad de la información.

A futuro, la cada vez y más acelerada expansión de los mercados y la continua necesidad de comunicaciones homogéneas entre cualquier tipo de organización sin duda llevarán a un proceso de implementación global de los estándares mencionados a lo largo de este texto, considerando que ello implica adquirir un mayor grado de especialización en el sentido de ser capaces de analizar y utilizar de manera asertiva la información que se genera mediante el uso de aplicativos y plataformas cada vez más sofisticadas y que exigen por parte de los profesionales contables un conjunto de habilidades que los pongan a la altura de la globalización a la que se están enfrentando.

Para la Superintendencia de Sociedades (2016) todo este proceso debe estar basado en el entendimiento de que se asiste a un nuevo contexto contable y financiero regulado por principios y no por normas en el que el enfoque de la información siempre ha sido y será la facilidad del usuario de la misma para estudiarla y obtener de ella cierta utilidad. Para ello, todo este conjunto de protocolos apunta a una mayor comparabilidad de la información, ya sea, por industria, sector o país.

\section{METODOLOGÍA}

El enfoque de este trabajo es cualitativo y estudia el caso del sector financiero colombiano en la implementación del lenguaje XBRL a través de la experiencia de una entidad significativa para este sector en el país. Como estrategia para la recolección de la información del caso, se documentó la regulación aplicable, así como la información que se documentó al interior de la entidad estudiada sobre el proceso que tuvieron que implementar para cumplir con este tipo de reporte.

Así mismo, se desarrolló una entrevista a una persona que directamente estuvo vinculada al proceso a quien se le preguntó lo siguiente:

¿Cuál es su área de desempeño en el banco?

¿Cuál fue su rol en el proceso de implementación de XBRL en el banco?

¿Hace cuánto tiempo iniciaron la preparación para esta implementación y cómo fue el proceso en la práctica?

¿Cuáles fueron las limitaciones o retos que enfrentaron en el proceso de implementación?

¿Qué ventajas observan en la implementación del XBRL para el reporte de información? 
¿Cuáles fueron los principales aprendizajes que quedaron para el banco durante este proceso?

¿Cómo fue el proceso de relacionamiento con la superintendencia financiera para cumplir con esta obligación?

¿Cuáles son las perspectivas del banco para el futuro sobre esta temática?

Una vez sistematizada la documentación sobre el caso y procesada la información de la entrevista obtenida, se procedió con el análisis de la información a luz de la reconstrucción de lo sucedido para esta entidad, que concuerda con lo vivido por el sector financiero colombiano hacia la implementación del lenguaje XBRL en sus reportes.

\section{IMPLEMENTACIÓN DEL XBRL EN COLOMBIA: EL CASO DEL SECTOR FINANCIERO}

La Superintendencia Financiera de Colombia, como ente de regulación local para las entidades del sector financiero, emitió las circulares externas 007 y 011 en 2015 encaminadas a la adopción de XBRL para el reporte de información de los estados financieros separados y consolidados y sus notas, tanto intermedios como de cierre de periodo. La intención de reportar información financiera bajo este lenguaje surgió en el marco de la implementación de NIIF en el sector; proceso que tenía por resultado la presentación por primera vez bajo estos estándares en ese mismo año.

Por lo anterior, la Superintendencia financiera inicialmente requirió a las entidades reportar desde el 2015 información bajo XBRL relacionada con los estados financieros (210000, 220000, 310000, 320000, 410000, 420000, 510000, 520000 y 610000), información general sobre los estados financieros (110000), comentarios de la gerencia (105000), adopción por primera vez (819100), información financiera intermedia (813000) e información de la entidad y declaración de cumplimiento con las NIIF (810000); y posteriormente en 2016, el reporte completo de estados financieros y notas en XBRL. No obstante, la complejidad de la implementación de este lenguaje de reporte, debido al desconocimiento generalizado y la transformación técnica de los procesos de revelación de información financiera requerida en las organizaciones, obligó al regulador a modificar el proyecto y trasladar el cumplimiento para el año 2016, por lo cual expidió la CE 038 de 2015 requiriendo a las entidades transitoriamente la presentación en formatos Excel (.xls) durante el primer año.

A partir de los pronunciamientos del regulador, la entidad objeto de estudio y las entidades financieras en general, emprendieron planes de implementación del lenguaje incluyendo el estudio del XBRL, la evaluación del impacto en los procesos internos relacionados con el reporte de información financiera y la adecuación de herramientas tecnológicas existentes, así como la integración a su estructura de nuevas aplicaciones.

La incorporación del reporte de información financiera en XBRL supuso un gran reto para todas las organizaciones. De un lado, las entidades que revelan información debieron realizar una exploración inicial y entendimiento estructural del lenguaje XML y en particular de la etiqueta de los datos para añadir descripciones y contextos (metadatos). Además de estructura y contenido de las revelaciones desde el punto de 
vista de NIIF y su diseño y representación en este lenguaje, considerando que para el 2015 las entidades aplicaban por primera vez los estándares internacionales.

Por otra parte, el regulador también ha realizado un esfuerzo notable, por impartir instrucciones relacionadas con el reporte de información que permitiera la utilización de dicha información financiera de manera comprensible, transparente y comparable para cualquier inversionista o usuario de dicha información a nivel nacional e internacional. (Superintendencia financiera de Colombia, 2015). La implementación de XBRL para el regulador implicó robustecer su plataforma de información y capacitarse en el lenguaje, no sólo por las diferencias tan significativas de los nuevos reportes en relación con los informes periódicos (diarios, semanales, mensuales, trimestrales, semestrales y anuales) en Excel, Word (.docx) o PDF que las entidades transmitían previamente, sino también porque esos medios antiguos de publicación de estados financieros se mantuvieron para convivir con las nuevas formas de reporte.

En septiembre de 2016, la entidad financiera objeto de estudio ya reportaba información de sus estados financieros condensados, completos, separados y consolidados completamente en XBRL al igual que otras entidades del sector financiero, lo cual significa que en menos de dos años se adoptó el lenguaje, estableciendo una diferencia significativa en relación con otros países de la región que también están migrando a este lenguaje. A continuación, se describen algunas experiencias en países y entidades a nivel mundial.

Caso mexicano: a partir de la adopción de NIIF por parte de las entidades cotizadas en la Bolsa Mexicana de Valores (BMV) en 2012, esta entidad asumió la responsabilidad de construir las taxonomías para las entidades públicas, por lo cual durante ese año y en 2013 se dedicó a estructurar inicialmente estados financieros con base en la taxonomía publicada por el IASB y en el catálogo contable local. Luego, en 2014 requirió a las entidades para presentar a través de un esquema inicial de taxonomía que permitiera identificar de manera temprana posibles errores en las etiquetas desarrolladas durante la etapa inicial del proceso. Sólo hasta 2015, la BMV en conjunto con la Comisión Nacional Bancaria y de Valores (CNBV) publicaron la versión final de las siete taxonomías creadas, que incluyen 17 informes sobre los estados financieros y notas que las entidades debían aplicar a partir de 2016 (Minaburo, 2015).

Caso SEC - Comisión de Bolsa y Valores (Estados Unidos): en conjunto con la Unión Europea, esta entidad después de analizar las ventajas y desventajas del XBRL, en el año 2004 empezó a adoptar voluntariamente el lenguaje, manifestando esto oficialmente al siguiente año donde empezó a realizar las pruebas pilotos a través de la plataforma EDGAR. Finalmente, en el año 2009 se evidenció la obligatoriedad de la aplicación del XBRL, por lo que las compañías de este país reportaron informes a la SEC. Con la aplicación voluntaria, la SEC quería comprobar de primera mano la efectividad del lenguaje en el reporte y comunicación de información a los diferentes usuarios.

Como se ha mencionado antes, el proceso de implementación de reporte en XBRL para las entidades financieras, ha sido notoriamente más acelerado que en otros casos de implementación observados en economías cercanas. A pesar de los esfuerzos indicados que han debido hacer las entidades, se podría cuestionar si efectivamente 
en Colombia se ha realizado un proceso de implementación adecuado en relación al tiempo que tanto el regulador como los emisores de información se han debido tomar para identificar posibles errores o mejoras en los desarrollos efectuados sobre la taxonomía, establecer criterios particulares de revelación con base en las características propias de las entidades del sector y de regulaciones específicas locales en materia contable que conviven con la aplicación de NIIF, tanto en estados financieros consolidados como separados.

\subsection{Requerimientos de la información en XBRL por la regulación local}

Desde la implementación de XBRL en el sector financiero, el regulador ha apuntado a la generación de informes de calidad que permitan la comparación con otras entidades del sector e incluso del mundo. En sus primeras disposiciones normativas requirió que la información reportada de fin de ejercicio fuese acompañada de la certificación del representante legal y del contador público y el dictamen del revisor fiscal o del auditor externo, en tanto que para estados financieros intermedios presentados de conformidad con NIC 34 - Estados financieros intermedios, sólo se exigió la inclusión de su firma digital. Lo anterior, delegó el compromiso y la responsabilidad a las entidades de construir y presentar información completa y de calidad y a las firmas auditoras a realizar trabajos de aseguramiento de dicha información desde el momento inicial de la implementación (Superintendencia financiera de Colombia, 2015).

El regulador ha requerido, además, una periodicidad trimestral en el reporte de información financiera intermedia que cumpla con todos los requerimientos de NIC 34 que contiene, entre otros, estados financieros condensados, notas seleccionadas con información relevante, segmentos de operación e información a revelar sobre el valor razonable de los instrumentos financieros. Las discrepancias entre las entidades financieras y el regulador se mantienen en la actualidad, ya que en las primeras, se considera que la medida es excesiva si se observa que la NIC 34 "aconseja a las entidades cuyos títulos cotizan en los mercados que... (a) publiquen, al menos, estados financieros intermedios referidos al primer semestre de cada uno de sus periodos contables anuales" (IFRS Foundation, 2000), sin embargo, no se presenta un requerimiento desde el estándar internacional que obligue a las entidades a realizar presentación alguna. En razón a esto, la Asobancaria, actualmente gestiona la disminución del volumen de información requerida ante la Superintendencia Financiera de Colombia.

\subsection{De la utilidad de la información financiera en $X B R L$}

La utilización del XBRL en el contexto organizacional puede generar grandes beneficios y ahorro de costos en la administración, reporte y análisis de información financiera y no financiera tanto interna de la organización, como aquella dispuesta para publicación de los diferentes usuarios externos a ellas. Debido a las cualidades de extensibilidad que posee, este lenguaje se prevé que pueda convertirse en una herramienta de intercambio de información que le otorgue a la entidad mayor agilidad en la generación de reportes, construcción de comparativos e inclusive, la disminución del riesgo de error o fraude en la información. A pesar de todas sus bondades, las entidades del sector financiero colombiano, y en especial la entidad financiera de estudio, aún distan de obtener todo el provecho posible del XBRL, dado que en la actualidad las taxonomías se 
encuentran restringidas en su extensibilidad por parte del ente regulador y esta forma de reporte confluye con otros documentos que históricamente se han transmitido a la Superfinanciera con diferentes periodicidades, como lo son:

- Saldos de estados financieros

- Informes financieros trimestrales y anuales

- Tasas de captación e indicadores de liquidez

- Encaje bancario

- Posición propia en moneda extranjera

- Tasas de captación

- Tasas y desembolsos por modalidad de créditos y libranzas

- Indicadores financieros bajo NIIF

- Portafolios de inversión

- Evolución de tarjetas de créditos

- Informes sobre mercados de divisas

Hasta la fecha, la presentación de información financiera por parte de las entidades del sector se ha enfocado en la construcción tradicional de sus formatos y luego, con base en ellos, se construye la información aplicable a XBRL. Particularmente, para los estados financieros de fin de ejercicio, los informes anuales sirven como base para la construcción de las instancias que finalmente son reportadas a Superintendencia Financiera y publicadas al mercado.

Esta dinámica observada ha hecho que el mercado en general no se haya interesado lo suficiente como para adoptar la información disponible en lenguaje XML (instancias publicadas) para realizar estudios del mercado, informarse sobre resultados y crear perspectivas financieras. En su lugar, han continuado utilizando los reportes en su forma histórica, impulsados quizá por la resistencia al cambio frente al nuevo lenguaje, la disponibilidad inmediata de los modelos que ya dominan y sobre los cuales se tienen estructurados andamiajes para la administración de la información que estos suministran, sobre todo en la construcción de información estadística.

En la entidad financiera objeto de estudio y en general, en el sector financiero colombiano, aún no se percibe el valor agregado que puede otorgar la utilización del XBRL como herramienta de intercambio de información, puesto que en la actualidad los reportes solo han sido considerados "de cumplimiento" y, por tanto, la interacción con esta información solo se limita a la transmisión al ente de control.

Es de esperarse, como suele suceder en las implementaciones de nuevos estándares o tecnologías, que poco a poco las entidades y los usuarios de la información externos obtengan un conocimiento más sólido sobre las bondades del XBRL y se comience a aprovechar de manera más proactiva la información disponible sobre la situación financiera y los rendimientos del sector en la toma de decisiones de inversión, principalmente. Por ahora, las entidades juegan un papel de cumplimiento y de construcción de información de calidad, con miras al suministro de información de calidad a los posibles usuarios interesados actuales y futuros a pesar de que en el corto plazo no se hayan identificado y experimentado claramente los beneficios de la utilización de este lenguaje. 


\section{CONSIDERACIONES FINALES}

A partir de lo observado en la entidad objeto de estudio, considerada como representativa en el sector financiero, se puede afirmar que apenas se tienen los primeros aprendizajes de la aplicación del XBRL. A pesar de reportar a Superintendencia Financiera un gran contenido de información bajo el nuevo marco técnico normativo contable que finalmente se pone a disposición del público, aún no se tiene claridad sobre el impacto que la utilización del lenguaje puede tener en el mercado y cómo esto facilitaría el acceso y administración de información, así como la toma de decisiones por parte de los distintos usuarios interesados.

Las entidades del sector tienen un largo camino por recorrer para lograr una implementación efectiva del XBRL en sus procesos contables. De momento, la construcción de información para efectos de este lenguaje es accesoria y posterior al ciclo contable del periodo, por tanto, en la medida que el XBRL pueda facilitar la interacción de información desde un momento más inicial del proceso, podrían empezar a hacerse más visibles sus beneficios en la organización.

El proceso de implementación del XBRL en el sector financiero, será referencia para la adopción en las demás entidades de la economía colombiana (entidades públicas y privadas), de modo que puedan aprovechar los beneficios que representa la aplicación de dicho mecanismo de comunicación para la presentación de la información contable y financiera. Así como la utilidad que presenta para los tomadores de decisiones y para la administración de la entidad en general.

\section{REFERENCIAS BIBLIOGRAFICAS}

AECA - Asociación Española de Contabilidad y Administración de Empresas. (2003). XBRL: un estándar para el Intercambio Electrónico de Información Económica y Financiera. Madrid: Asociación Española de Contabilidad y Administración de Empresas.

Asobancaria. (2012). Convergencia a NIIF para el sector bancario. Obtenido de Instituto Nacional de Contadores Públicos: http://www.incp.org.co/ incp/document/convergencia-a-niif-para-el-sector-bancario/

Caballero, C. (2010). Un rápido recorrido por la historia del sector financiero en Colombia. Obtenido de Revista Dinero: http://www.dinero.com/ columnistas/edicion-impresa/articulo/un-rapido-recorrido-historiadel-sector-financiero-colombia-carlos-caballero-argaez/95393

Cano, A. (2009). Las normas internacionales en la contabilidad y la entrada en vigencia de la ley 1314 de 2009 en el contexto contable colombiano. Recuperado el agosto de 2013, de Observatorio de la Economía Latinoamericana: http://www.eumed.net/cursecon/ecolat/co/09/amcm.htm

Castaño, C. E. (2016). Reflexiones sobre la investigación contable en Colombia. Panorama Económico, 24, 163-174. 
Consejo Técnico de la Contaduría Pública CTCP. (2016). Documento de Sustentación de la Propuesta a los Ministerios de Hacienda y Crédito Público y de Comercio, Industria y Turismo sobre la aplicación de las Normas Internacionales de Información Financiera en Colombia. Obtenido de Ministerio de Hacienda y Crédito Público: http://www.minhacienda. gov.co/HomeMinhacienda/ShowProperty?nodeId=\%2FOCS\%2FP_ MHCP_WCC-061427\%2F\%2FidcPrimaryFile\&revision=latestreleased

DANE. (2017). Boletín Técnico: Producto Interno Bruto (PIB). Obtenido de Sitio web del DANE: http://www.dane.gov.co/files/investigaciones/boletines/ pib/bol_PIB_IVtrim16_oferta_demanda.pdf

Debreceny, Felden, Ochocki \& Piechocki. (2009). XBRL for interactive data. New York: Springer-Verlag Berlin Heidelberg.

Hernández, R. (2017). El sistema financiero, el sector de mayor crecimiento en los últimos años. Obtenido de El Heraldo: https://www.elheraldo. $\mathrm{co} / \mathrm{mas}$-negocios/el-sistema-financiero-el-sector-de-mayorcrecimiento-en-los-ultimos-anos-323885

IFRS Foundation. (2000). NIC 34 Información Financiera Intermedia. NIC 34 Información Financiera Intermedia. IFRS Foundation.

Jensen, R., \& Xiao, J. (2001). Customized financial reporting, networked databases, and distributed file sharing. Accounting Horizons, 15(2), 209-222.

LEGIS. (2014). ABC del XBRL en Colombia. Obtenido de Legis, Comunidad Contable: http://www.comunidadcontable.com/BancoConocimiento/N/ noti-0701201401_\%28abc_del_xbrl_en_colombia\%29/noti0701201401 \%28abc_del_xbrl_en_colombia\%29.asp

López, C., \& Zea, F. (2011). Convergencia a estándares internacionales en Colombia: análisis de documentos del CTCP. Legis, 98-134.

Minaburo, S. (2015). Dirección estratégica. La revista de negocios del ITAM. Obtenido de Dirección estratégica: http://direccionestrategica.itam.mx/ xbrl-su-desarrollo-y-aplicacion-en-mexico/

Mishkin, F. (1996). Understanding Financial Crises: A Developing Country Perspective. Obtenido de The National Bureau of Economic Research: http://www.nber.org/papers/w5600

Ocampo, J. (2015). Una historia del sistema financiero colombiano. Tiempo \& Economía, 2(2), 115-121.

Publicaciones Semana. (2015). Las 7 principales amenazas al sistema financiero en 2015. Obtenido de Revista Dinero: http://www.dinero.com/economia/ articulo/los-sriesgos-para-sisistema-financiero-2015/205021 
Santos, E. (2005). La Misión Kemmerer. Obtenido de Biblioteca Virtual del Banco de la República de Colombia: http://www.banrepcultural.org/ blaavirtual/revistas/credencial/abril2005/mision.htm

Superintendencia de Sociedades. (2016). SIRFIN, Sistema Integrado de Reportes Financieros. Obtenido de Formación XBRL: http://superwas. supersociedades.gov.co/sirfin/Manual_de_funcionamiento_SIRFIN.pdf

Superintendencia de Sociedades. (2015). Superfinanciera. Obtenido de Superfinanciera: https://www.superfinanciera.gov.co/descargas?com =institucional\&name=pubFile1012583\&downloadname=ce007_ 15.doc_

Superintendencia Financiera de Colombia. (2015). Taxonomías 2015. Obtenido de Documentos XBRL Superfinanciera: https://www.superfinanciera.gov.co/jsp loader.jsf?1Servicio=Publicaciones\&1Tipo=publicacion\&1Funcion=load ContenidoPublicacion\&id=10084416

Zamarra, J.; Atehortúa, T., Sierra, V. (2017). Homogenización y comparabilidad de la información financiera a través del XBRL. Contaduría Universidad de Antioquia, 71, 213-231. 